\title{
Letramento funcional em saúde: sífilis em gestantes
}

RESUMO | Este trabalho teve como objetivo mensurar o nível de letramento funcional em saúde e o nível de conhecimento sobre sífilis em um grupo de gestantes. Foi utilizado questionário sociodemográfico, a versão brasileira do B-THOFLA (Brief Test of Functional Health Literacy in Adults) para mensurar o letramento funcional em saúde e questionário com perguntas básicas sobre sífilis. Obteve-se como resultado: maior parte do grupo tendo um perfil jovem e possuindo até 25 anos, tendo alto índice da não utilização de preservativos, um bom letramento funcional em saúde e um baixo nível de conhecimento sobre sífilis. Se caracterizando como um grupo altamente vulnerável a infecções sexualmente transmissíveis, incluindo a sífilis.

Palavras-chaves: Letramento Funcional em Saúde; Sífilis; Gestantes.

ABSTRACT | This study aimed to measure the level of functional health literacy and the level of knowledge about syphilis in a group of pregnant women. A sociodemographic questionnaire, the Brazilian version of the B-THOFLA (Brief Test of Functional Health Literacy in Adults) was used to measure functional health literacy and a questionnaire with basic questions about syphilis. As a result, most of the group had a young profile and were up to 25 years old, with a high rate of non-use of condoms, good functional health literacy and a low level of knowledge about syphilis. Characterizing as a highly vulnerable group to sexually transmitted infections, including syphilis.

Keywords: Functional Health Literacy; Syphilis; Pregnant Women.

RESUMEN | Este estudio tuvo como objetivo medir el nivel de alfabetización funcional en salud y el nivel de conocimiento sobre la sífilis en un grupo de mujeres embarazadas. Se utilizó un cuestionario sociodemográfico, la versión brasileña de B-THOFLA (Brief Test of Functional Health Literacy in Adults) medir la alfabetización funcional en salud y un cuestionario con preguntas básicas sobre la sífilis. Como resultado, se obtuvo: la mayoría del grupo tenía un perfil joven y hasta 25 años, con una alta tasa de no uso de condones, buena alfabetización funcional en salud y un bajo nivel de conocimiento sobre la sífilis. Se caracteriza por ser un grupo altamente vulnerable a las infecciones de transmisión sexual, incluida la sífilis.

Palavras claves: Alfabetización Funcional en Salud; Sífilis; El embarazo.

\section{Claudia Curbani Vieira Manola}

Enfermeira. Mestre em Administração. Centro Universitário Salesiano.

\section{Evandro Bernardino Mendes de Melo}

Enfermeiro. Mestre em Saúde Coletiva. Centro Universitário Salesiano.

\section{Yhago Kauan Correia Lau}

Enfermeiro. Centro Universitário Salesiano.

\section{Lívia Perasol Bedin}

Enfermeira. Doutora em Educação. Centro Universitário Salesiano.

\section{Maristela Vilarinho de Oliveira}

Enfermeira. Especialista em Administração de Recursos Humanos. Centro Universitário Salesiano.

\section{Miriam Aparecida Inácio de Almeida}

Estatística. Mestre em Engenharia Ambiental. Centro Universitário Salesiano.

\section{Magda Ribeiro de Castro}

Enfermeira. Doutora em Enfermagem. Universidade Federal do Espirito Santo.

\section{Priscilla Silva Machado}

Enfermeira. Doutora em Enfermagem. Universidade Federal do Espirito Santo

Recebido em: 23/05/2020

Aprovado em: 23/05/2020
INTRODUÇÃO

egundo o Ministério da Saúde ${ }^{(1)}$,

sífilis é uma das infecções sexualmente transmissíveis que teve sua taxa de incidência elevada nos últimos anos no território nacional. Sendo uma dessas complicações a sífilis em gestantes, que pode levar ao desenvolvimento da sífilis congênita, visto que esses dois tipos de manifestação da doença também cresceram em números de casos. Paradoxalmente, mesmo com as altas taxas de incidência, a sífilis pode ser controlada, levando-se em consideração a existência de testes diagnósticos sensíveis, tratamento eficiente e de baixo ônus.

É mais eficiente combater através de prevenção, campanhas focadas no público-alvo e promoção. Existem fatores que necessitam ser considerados, como: baixa renda, nível de escolaridade, estado civil entre outros. Letramento funcional em saúde é a competência cognitiva de entender, interpretar e aplicar informações escritas ou verbalizadas sobre saúde(2).

O letramento em saúde é importante para o autocuidado de pacientes, todavia também os afeta desde a prevenção e diagnóstico até o compreendimento da doença e seu tratamento ${ }^{(3,4)}$. Uma das principais funções do enfermeiro é de educar. A educação em saúde se consiste em um instrumento fundamental para uma assistência de boa qualidade, através do qual o enfermeiro faz orientações para o autocuidado, tanto em nível individual até em nível coletivo, transformando indivíduos, famílias e comunidades em multiplicadores de conhecimentos em saúde ${ }^{(5)}$.

Há uma dificuldade quando se trata de prevenir a transmissão vertical, desde estabelecer um diagnóstico precoce até o tratamento adequado. Entende-se como tratamento adequado à gestante, quando se é utilizada penicilina e que tenha chegado ao termino de um mês antes do nascimento da prole. Estando a dose da medicação de acordo com o estágio da sífilis e o parceiro também sendo tratado ao mesmo tempo. Através de uma união de esforços entre pré-natal de boa qualidade, exames laboratoriais em adequado tempo, tratamento dos indivíduos que se 
relacionaram sexualmente e a conscientização de todos, poderemos conseguir controlar essa infecção ${ }^{(6)}$.

Diante da realidade descrita, o objetivo deste estudo consiste em avaliar o nível de letramento em saúde assim como o conhecimento relacionado à sífilis. Como objetivo específico, conhecer letramento em saúde, discutir educação em saúde e correlacionar o nível de letramento com conhecimento da Sífilis.

\section{METODOLOGIA}

Trata-se de um estudo transversal e quantitativo que foi realizado nos meses de outubro e novembro de 2019.

Os locais para realização do estudo foram postos de saúde e centros comunitários onde havia ações da extensão universitária "Bem Nascer" da Católica de Vitória Centro Universitário. Nessa extensão universitária, é trabalhado com gestantes e acompanhantes educação em saúde voltada para a saúde da mulher e gestação, autocuidado, infecções sexualmente transmissíveis, amamentação, parto, alterações no corpo da gestante, que acontecia em postos de saúde e centros comunitários da Grande Vitória no Estado do Espírito Santo.

A população do estudo foi de 30 gestantes, independente do período gestacional, com idade igual ou maior que 18 anos, capazes de entender os objetivos do estudo e que sabiam ler e escrever, além de participarem da extensão universitária "Bem Nascer".

A coleta de dados se deu por meio da aplicação de questionário sociodemográfico que também possuía oito perguntas sobre sífilis e instrumento de avaliação de letramento funcional em saúde. As participantes foram abordadas nas reuniões da extensão universitária e foi explicado sobre o estudo e seus objetivos. Depois de acordado e assinado o Termo de Consentimento Livre e Esclarecido (TCLE), elas responderam aos questionários.

Primeiro, foram respondidos o questionário sociodemográfico e as questões básicas sobre sífilis, logo após, o instrumento para avaliar letramento em saúde, sendo este último possuindo um tempo máximo para resposta de 3 minutos. Não foi avisado de antemão sobre o tempo de duração, quando findado o tempo era recolhido para posterior avaliação.

Os aspectos sociodemográficos presentes no estudo foram: idade, escolari-

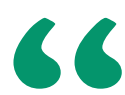

apenas de sífilis, mas de outras infecções sexualmente transmissíveis.

Foi utilizada a versão brasileira do B-THOFLA (Brief Test of Functional Health Literacy in Adults) para mensurar o letramento funcional em saúde que contém 19 itens de compreensão textual, sendo que cada item tem o valor de 1 ponto, dessa forma, a pontuação máxima é de 19 pontos. Foi considerado como nível de letramento funcional em saúde inadequado a pontuação de 0 a dez pontos; médio letramento funcional em saúde de 11 a 12 pontos; adequado letramento funcional em saúde de 13 a 19 pontos. Foi considerado adequado nível de letramento funcional em saúde o nível suficiente, e inadequado os níveis médio e baixo.

A avalição dos conhecimentos sobre sífilis foi realizada através de questionário contendo 8 questões básicas sobre a enfermidade. Cada pergunta tinha apenas uma resposta certa, a pontuação total se dá pela soma da quantidade de perguntas respondidas de forma correta, cada questão possuía o valor de 1 ponto. Foi considerado nível de conhecimento baixo sobre sífilis quando atingido no máximo o percentil 59 (0-4 pontos); médio conhecimento sobre sífilis entre os percentis 60 e 74 (5 pontos); suficiente conhecimento sobre sífilis igual ou acima do percentil 75 (6-8 pontos). Foi estipulado como adequado conhecimento sobre sífilis o nível suficiente, e inadequado os níveis médio e baixo.

A análise realizada neste trabalho consiste na exploração dos dados utilizando estatística descritiva (distribuição de frequências, gráficos e medidas de tendência central e variabilidade). Foi utilizado o programa computacional Micro-

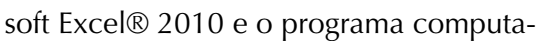
cional SPSS ${ }^{2} 23.00$ for Windows.

Este trabalho foi aprovado pelo Comitê de Ética e Pesquisa da Católica e Vitória Centro Universitário sob o parecer de n. ${ }^{\circ}$ 3.707.458. Tendo todas as participantes assinado o TCLE antes de executado o protocolo de estudo. 
Tabela 1. Caracteristicas sociodemográficas de $\mathbf{3 0}$ gestantes participantes. Vitória, ES, Brasil, 2019.

\begin{tabular}{|cc|}
\hline Caracteristicas & $\mathbf{n}(\%)$ \\
\hline Idade & $7(23,3)$ \\
\hline 18 anos & $20(66,7)$ \\
\hline 19 a 25 anos & $3(10,0)$ \\
\hline 26 a 40 anos & \\
\hline Escolaridade & $3(10,0)$ \\
\hline Fundamental Incompleto & $5(16,7)$ \\
\hline Fundamental Completo & $6(20,0)$ \\
\hline Médio Incompleto & $12(40,0)$ \\
\hline Médio Completo & $3(10,0)$ \\
\hline Superior Incompleto & $1(3,3)$ \\
\hline Superior Completo & \\
\hline Estado Civil & $10(33,3)$ \\
\hline Solteiro (a) & $11(36,7)$ \\
\hline Casado (a) & $9(30,0)$ \\
\hline União Estável & \\
\hline Quantidade de filhos & $10(33,3)$ \\
Nenhum & $4(13,3)$ \\
Um & $9(30,0)$ \\
Dois & $4(13,3)$ \\
Três & $2(6,7)$ \\
\hline Quatro & $1(3,3)$ \\
\hline Cinco & \\
\hline
\end{tabular}

Tabela 2. Distribuição de Frequências segundo Gestação. Vitória, ES, Brasil, 2019.

\begin{tabular}{ccc} 
Gestação & Frequência & $\%$ \\
\hline Gestando pela $1^{\text {a }}$ vez & 6 & 20,0 \\
\hline 2 a 4 & 24 & 80,0 \\
\hline Total & 30 & 100,0 \\
\hline
\end{tabular}

Tabela 3. Distribuição de Frequências segundo Aborto. Vitória, ES, Brasil, 2019.

\begin{tabular}{ccc} 
Aborto & Frequência & $\%$ \\
\hline Nenhum & 15 & 50,0 \\
\hline Um & 5 & 16,7 \\
\hline Dois & 6 & 20,0 \\
\hline Três & 3 & 10,0 \\
\hline Quatro & 1 & 3,3 \\
\hline Total & 30 & 100,0 \\
\hline
\end{tabular}

\section{RESULTADOS E DISCUSSÃO}

Todas as participantes eram do sexo feminino, gestantes e habitavam na região metropolitana do estado do Espírito Santo. Sendo em sua maioria com idade entre 18 e 25 anos (90\%), com até o ensino médio completo $(86,7 \%)$, casadas e com união estável $(66,7 \%)$, possuindo até dois filhos $(76,6 \%)$.

Mesmo o grupo de estudado possuir um perfil jovem, mulheres com até 25 anos em sua maioria, cerca de $80 \%$ das participantes estavam, quando a pesquisa foi feita, gestando pelo menos pela segunda vez (Tabela 2). Contrastando também com esse perfil jovem está a alta incidência de abortamento, onde metade do grupo já teve no mínimo um aborto (Tabela 3 ).

Segundo dados da Tabela 4, que diz respeito a autoafirmações a respeito da vida sexual, foi constatado que a maior parte do grupo que respondeu à questão sobre a quantidade de relações sexuais desprotegidas que já teve na vida teve mais de 21 relações sexuais desprotegidas, representando $43,3 \%$ do grupo.

Cerca de mais da metade da amostra refere não utilizar nenhum método contraceptivo, aproximadamente um terço usa anticoncepcional oral ou injetável e um décimo diz usar tabelinha. O único método contraceptivo que é eficaz na prevenção de infecções sexualmente transmissíveis é o preservativo masculino/feminino, todavia as participantes não referiram utilizar em suas relações, demonstrando uma vulnerabilidade a possível infecção não apenas de sífilis, mas de outras doenças também (Tabela 5). Por ser uma doença de transmissão sexual, entende-se que quanto mais relações desprotegidas o indivíduo tiver, maiores serão suas chances de contrair a doença. Sendo a sífilis apenas uma das várias doenças e in- 
Tabela 4. Distribuição de Frequências segundo Quantidade de relações sexuais desprotegidas. Vitória, ES, Brasil, 2019.

\begin{tabular}{|ccc|}
\hline Quantidade de relações sexuais desprotegidas & Frequência & $\%$ \\
\hline 1 & 4 & 13,3 \\
\hline De 2 a 5 & 7 & 23,3 \\
\hline De 6 a 10 & 1 & 3,3 \\
\hline De 11 a 20 & 1 & 3,3 \\
\hline Mais de 21 & 13 & 43,3 \\
\hline Não respondeu & 4 & 13,3 \\
\hline Total & 30 & 100 \\
\hline
\end{tabular}

Tabela 5. Distribuição de Frequências segundo Método Contraceptivo. Vitória, ES, Brasil, 2019.

\begin{tabular}{ccc} 
Método Contraceptivo & Frequência & $\%$ \\
Não utiliza & 16 & 53,3 \\
\hline Anticoncepcional oral/injetável/ adesivo & 10 & 33,3 \\
Tabela & 3 & 10 \\
Não respondeu & 1 & 3,3 \\
Total & 30 & 100
\end{tabular}

Tabela 6. Distribuição de Frequências segundo Onde recebeu informação sobre sifilis. Vitória, ES, Brasil, 2019.

$\begin{array}{ccc}\text { Onde recebeu informação sobre sífilis } & \text { Frequência } & \% \\ \text { Nunca recebi } & 4 & 13,3 \\ \text { Pré-natal } & 10 & 33,3 \\ \text { Escola } & 9 & 30,0 \\ \text { Amigos } & 1 & 3,3 \\ \text { TV } & 1 & 3,3 \\ \text { Livros } & 1 & 3,3 \\ \text { Outros } & 1 & 3,3 \\ \text { Não respondeu } & 3 & 10,0 \\ \text { Total } & 30 & 100,0\end{array}$

Tabela 7. Proporção de acertos e erros dos itens relacionados ao nível de conhecimento da sífilis, respondidos por 30 pacientes. Vitória, ES, Brasil, 2019.

\begin{tabular}{ccc|}
\hline Questões referentes ao Nível de Conhecimento da Sífilis & Acertos $\mathbf{n}(\%)$ & Erros $\mathbf{n}(\%)$ \\
\hline Como se pega a sífilis & $26(86,7)$ & $4(13,3)$ \\
\hline Quais os sintomas da sífilis & $8(26,7)$ & $22(73,3)$ \\
\hline Sífilis tem cura & $18(60,0)$ & $12(40,0)$ \\
\hline É possível passar sífilis para o filho quando está grávida & $18(60,0)$ & $12(40,0)$ \\
\hline É possivel ter sífilis quantas vezes & $11(36,7 \%)$ & $19(63,3)$ \\
\hline Quais os perigos de transmitir sífilis para o bebê & $16(53,3)$ & $14(46,7)$ \\
\hline Mãe com sífilis pode amamentar & $7(23,3)$ & $23(76,7)$ \\
\hline Tratamento indicado para a sífilis & $13(43,3)$ & $17(56,7)$
\end{tabular}

fecções sexualmente transmissíveis. Segundo estudo(7), haverá redução de novos casos de infecções sexualmente transmissíveis quando houver mudanças em como as pessoas se comportam sexualmente, principalmente se utilizarem de maneira adequada o preservativo. Essas mudanças ocorrem através de incessante informação ao público geral e ações educativas que promovam o reconhecimento de riscos e promoção de medidas que visam a prevenção.

Relacionado sobre o recebimento de informações sobre sífilis, aproximadamente $1 / 3$ diz ter recebido informações no pré-natal. Não foi questionada nesse estudo a adesão dessas gestantes à assistência pré-natal, visto que o "Bem Nascer" também realizava suas ações em reuniões coletivas de pré-natal em Unidades Básicas de Saúde. Apesar de todas as participantes estarem gestantes, uma parcela pequena teve informações sobre sífilis na assistência pré-natal, segundo estudo(8), o Brasil possui elevada cobertura de assistência pré-natal, porém baixa adequação da assistência e, de acordo com outro estudo ${ }^{(9)}$, a assistência pré-natal oferecida pelo Sistema Único de Saúde não é totalmente eficaz. Pesquisa(10) diz que, mesmo as mulheres tendo recebido informações no pré-natal, é necessário melhorar a forma como é instituído o processo de comunicação, a fim de melhorar o cuidado e minimizar os riscos. Referente a ter recebido informações sobre a enfermidade na escola representa $30 \%$, mostrando a importância do ensino sobre saúde sexual nas instituições de ensino; $13,3 \%$ diz nunca ter recebido informações sobre sífilis, revelando que apesar do aumento no número de casos ainda há uma parte da população que desconhece sobre a doença (Tabela 6).

A análise da proporção de acertos e 
erros relacionados ao questionário sobre conhecimentos a respeito da sífilis indicou que a questão de maior acerto é referente a forma de contágio da sífilis. A maioria entende que a relação sexual é uma das principais formas de transmissão, mas apenas 26,7\% acertou a pergunta que questionava sobre os sintomas, mostrando a carência de conhecimentos necessários para a auto

\section{Figura 1. Classificação do Nivel de Conhecimento da Sifilis. Vitória, ES, Brasil, 2019.}

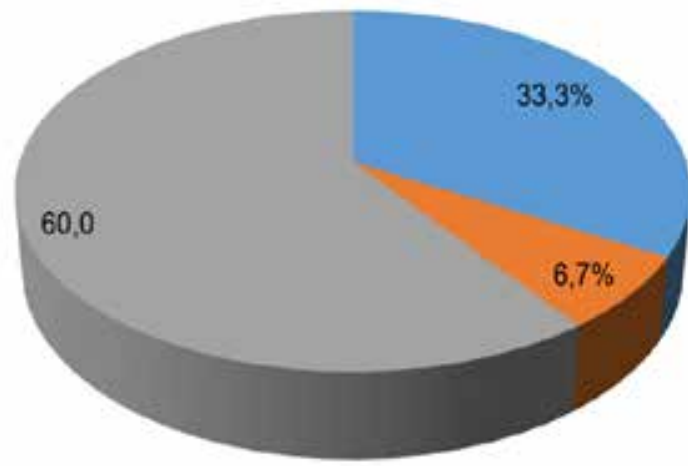

Suficiente

- Médio

Baixo

Figura 2. Classificação do Letramento. Vitória, ES, Brasil, 2019.

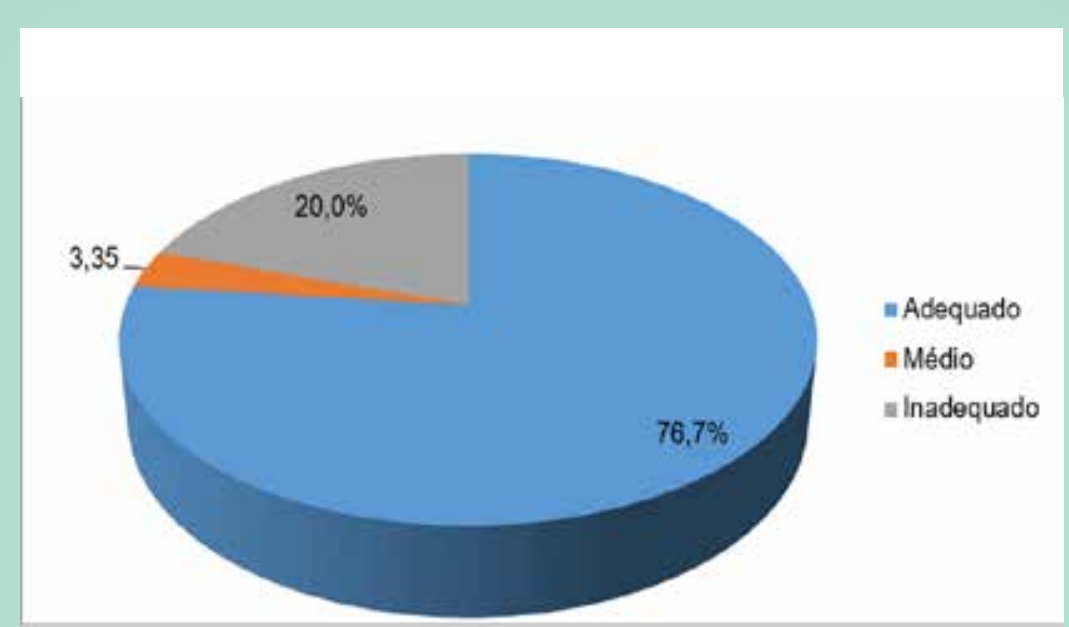

gestantes é uma importante ferramenta para a prevenção e promoção da saúde, desta forma, a gestante informada de forma correta se torna capaz de realizar escolhas que visam a não infecção, própria e da prole, além de se tornar uma possível disseminadora do conhecimento para $\mathrm{o}(\mathrm{s})$ parceiro(s) e outras gestantes.

Segundo o gráfico da Figura 1, a maior parte da amostra demonstrou ter um conhecimento insuficiente sobre a sífilis, apenas $1 / 3$ alcançou o nível considerado suficiente. Isso revela que os mecanismos de prevenção da sífilis não são suficientes e/ ou eficientes em passar para a população conhecimentos a respeito da doença, sendo as gestantes importante público-alvo, visto que, além do risco de contrair a sífilis adquirida, podem através da placenta infectarem sua prole, levando ao desenvolvimento da sífilis congênita quando não tratadas corretamente.

Estudo $^{(12)}$ relata que quanto maior o nível de conhecimento sobre a doença, maior é a preocupação em se proteger dela. Ações de educação em saúde se configuram em uma forma para a mudança desse cenário, onde a falta de conhecimento adequado é uma das principais causas para a vulnerabilidade dessas mulheres.

Existem poucos trabalhos nacionais sobre letramento funcional em saúde e que relacionam o nível de letramento funcional em saúde com o nível de conhecimento sobre sífilis. De acordo com a Figura 2, que ilustra a classificação do letramento funcional em saúde, o grupo, em sua maioria, apresentou adequado letramento em saúde, representando um total de $76,7 \%$ da amostra, contrastando com o nível de conhecimento sobre sífilis, onde a maioria não obteve rendimento satisfatório.

Os resultados demonstram que existe uma baixa na qualidade e quantidade de informações a respeito 
da sífilis nas gestantes participantes do estudo, revelando uma vulnerabilidade. Estudo(13) diz que intervenções educacionais baseadas em informações precisas e suficientes aos usuários dos serviços de saúde podem tornar melhores os resultados relacionados à saúde, despertando o interesse de forma ativa nos indivíduos com o intuito de desenvolverem o autocuidado em saúde. Nesse contexto, é essencial o papel da enfermagem em passar informações de forma clara, precisa, em linguajar acessível ao entendimento do sujeito e utilizando métodos necessários para a efetivação da educação em saúde de forma eficiente. A enfermagem detém capacidade e habilidades suficientes para realizar o cuidado através da educação em saúde ${ }^{(14)}$.

As limitações deste estudo estão relacionadas ao baixo volume de gestantes participantes, à pequena quantidade de estudos sobre letramento em gestantes e ao nível de conhecimento de gestantes sobre a sífilis, e a relação entre essas variáveis.

\section{CONCLUSÃO}

Este estudo teve como resultado
6

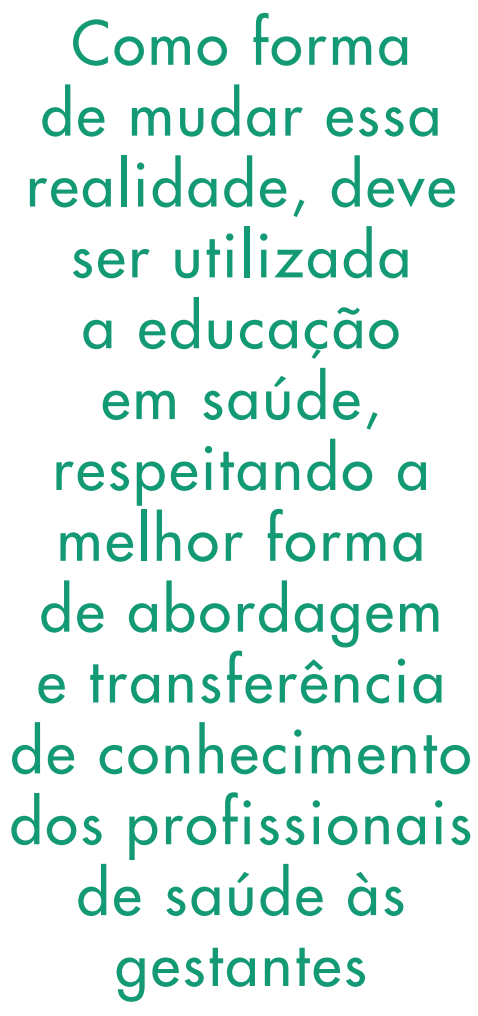

níveis de letramento funcional em saúde considerados adequados para a maioria da população estudada, e nível de conhecimento sobre sífilis inadequado para a maioria do grupo estudado, configurando-se uma não relação entre os níveis de letramento funcional em saúde e conhecimentos sobre sífilis em gestantes. Como forma de mudar essa realidade, deve ser utilizada a educação em saúde, respeitando a melhor forma de abordagem e transferência de conhecimento dos profissionais de saúde às gestantes, referindo desde o agente causador e sintomas, até consequências e limitações causadas pela infecção. Além de se enfatizar o uso de preservativos.

Sugere-se foco em ações de conscientização, prevenção e promoção a saúde com ênfase nas infecções sexualmente transmissíveis, especialmente em relação às sífilis adquirida e congênita, em mulheres que desejam ser mães e aquelas que estão em gestação. A educação em saúde com base na identificação do letramento auxiliaria na melhor forma de comunicação, pois estaria baseada na compreensão em saúde da comunidade.

\section{Referências}

1. Ministério da Saúde (BR). Boletim Epidemiológico [Internet]. Brasília (DF): MS, 2017 [acesso em 16 mai 2019]; 48(36):5-41. Disponível em: http://portalarquivos.saude.gov.br/images/pdf/2017/novembro/13/BE-2017-038-Boletim-Sifilis-11-2017-publicacao-.pdf.

2. Macêdo VC, Lira PIC, Frias PG, Romaguera LMD, Caires SFF, Ximenes RAA. Risk factors for syphilis in women: case-control study. Rev Saúde Pública. 2017; 51:78.

3. Adams RJ, et al. Health literacy: a new concept for general practice? Aust. Fam. Physician. 2009; 38(3):144-7.

4. Sampaio HAC, et al. Letramento em saúde de diabéticos tipo 2: fatores associados e controle glicêmico. Ciência \& Saúde Coletiva. 2015; 20:865-874.

5. Andrade $\mathrm{ME}$, et al. Percepção do enfermeiro quanto à sua atuação educativa na estratégia saúde da família. Rev Enferm UERJ. 2016; 1(5). 6. Lafetá KRG, et al. Sífilis materna e congênita, subnotificação e difícil controle. Revista Brasileira de Epidemiologia. 2016; 19:63-74.

7. Thiesen F. ISTs e uso de preservativos. Revista UNIPLAC. 2018; 6(1).

8. Viellas EF, et al. Assistência pré-natal no Brasil. Cadernos de Saúde
Pública. 2014; 30:S85-S100.

9. Domingues RMSM, et al. Sífilis congênita: evento sentinela da qualidade da assistência pré-natal. Revista de Saúde Pública. 2013; 47:147157.

10. Silva NM, et al. Conhecimento de puérperas sobre amamentação exclusiva. Rev Bras Enferm. 2014; 67(2):290-295.

11. Lima GK, et al. Educação em saúde sobre sífilis com um grupo de gestantes: um relato de experiência de acadêmicas de enfermagem. SANARE-Revista de Políticas Públicas, 2013; 12(2).

12. Costa TS, et al. Escola, sexualidade, práticas sexuais e vulnerabilidades para as infecções sexualmente transmissíveis (IST). Revista interdisciplinar de ensino, pesquisa e extensão. 2017; 4(1).

13. Oliveira GM, et al. Identificação do letramento em saúde para organização de atividades de educação em saúde com gestantes. 2019. $34 \mathrm{f}$ Trabalho de Conclusão de Residência (Atenção à Saúde Coletiva) - Universidade Federal de Uberlândia, Uberlândia, 2019.

14. Guerreiro EM, et al. Educação em saúde no ciclo gravídico-puerperal: sentidos atribuídos por puérperas. Rev bras enferm. 2014; 67(1):13-21. 